\title{
Beam normal spin asymmetry in the quasireal Compton scattering approximation
}

\author{
M. Gorchtein \\ Genoa University, Department of Physics, I-16146 Genoa, Italy \\ and California Institute of Technology, Pasadena, California 91125, USA* \\ (Received 10 May 2005; revised manuscript received 21 March 2006; published 1 May 2006)
}

\begin{abstract}
The two-photon exchange contribution to the single spin asymmetries with the spin orientation normal to the reaction plane is discussed for elastic electron-proton scattering in the equivalent photon approximation. In this case, the hadronic part of the two-photon exchange amplitude describes real Compton scattering (RCS). We show that in the case of the beam normal spin asymmetry this approximation selects only the photon helicity flip amplitudes of RCS. At low energies, we make use of unitarity and estimate the contribution of the $\pi N$ multipoles to the photon helicity flip amplitudes. In the Regge regime, the quasi-RCS (QRCS) approximation allows for a contribution from two-pion exchange, and we provide an estimate of such contributions.
\end{abstract}

DOI: 10.1103/PhysRevC.73.055201

PACS number(s): 13.60.Fz, 12.40.Nn, 13.40.Gp, 14.20.Dh

\section{INTRODUCTION}

Recently, the new polarization transfer data for the electromagnetic form factors ratio $G_{E} / G_{M}$ [1] raised a lot of interest for the two-photon exchange (TPE) physics in elastic electron proton scattering. These new data appeared to be incompatible with the Rosenbluth data [2]. A possible way to reconcile the two data sets was proposed [3], which consists of a more precise account of the TPE amplitude, the real part of which enters the radiative corrections to the cross section (Rosenbluth) and the polarization cross section ratio in a different manner. At present, only the IR divergent part of the two-photon exchange contribution, corresponding to one of the exchanged photon's being soft, is included in the experimental analysis [4]. Two model calculations exist for the real part of the TPE amplitude [5,6], and they qualitatively confirm that the exchange of two hard photons may be responsible for this discrepancy. To extract the electric form factor in a model-independent way, one thus has to study the general case of Compton scattering with two spacelike photons. These two-photon contributions are important for the electroweak sector, as well.

In view of this interest, parity-conserving single spin asymmetries in elastic $e p$ scattering with the spin orientation normal to the reaction plane regain attention. These observables are directly related to the imaginary part of the TPE amplitude and have been an object of theoretical studies in the 1960's and 1970's [7]. By analyticity, the real part of the TPE amplitude is given by a dispersion integral over its imaginary part. Therefore a good understanding of this class of observables is absolutely necessary. Recently, first measurements of the beam normal spin asymmetry ${ }^{1} B_{n}$ have been performed in different kinematics [8].

Though small (several to tens of parts per million), this asymmetry can be measured with a precision of fractions of parts per million. Before implementing different models for

\footnotetext{
*Electronic address: gorshtey@caltech.edu

${ }^{1}$ In the literature, also, the $A_{n}$ notation for beam normal spin asymmetry or vector analyzing power was adopted.
}

the real part, where an additional uncertainty comes from the dispersion integral over the imaginary part, one should check the level of understanding of the imaginary part of TPE. These checks have been done for the existing data. Inclusion of the elastic (nucleon) intermediate state only [9] led to negative asymmetry of several parts per million in the kinematics of SAMPLE experiment but was not enough to describe the data. The description of the beam normal spin asymmetry within a phenomenological model which uses the full set of the single-pion electroproduction [10] did not give satisfactory description at any of the available kinematics. Especially intriguing appears the situation with the SAMPLE data with electron lab energy $E_{\text {lab }}=200 \mathrm{MeV}$, which is just above the pion production threshold where the theoretical input is well understood. On the other hand, an EFT calculation without dynamical pions [11] was somewhat surprisingly very successful in describing this kinematics for $B_{n}$. This success suggests that to the given order of chiral perturbation theory the role of the dynamical pions for this observable might be not too large. Finally, a recent calculation within the leading logarithm approximation appeared, where only few dominant multipoles were used as input [12]. Surprisingly, the authors of Ref. [12] were able to describe all the experimental data at very different beam energies and scattering angles, while the full calculation of Ref. [10] failed to describe any of them.

Though even at low energies the situation with the imaginary part of TPE amplitude is far from clear, attention has to be paid to high energies, as well, since the dispersion integral that would give us its real part should be performed over the full energy range. Owing to the relative ease of measuring $B_{n}$ within the framework of parity-violating electron scattering, new data from running and upcoming experiments [13] will stimulate further theoretical investigations of this new observable. A calculation of $B_{n}$ in the hard kinematics regime at high energy and momentum transfer was performed recently in the framework of generalized parton distributions (GPD's) and resulted in asymmetries of $\sim 1.5 \mathrm{ppm}$ [14].

Since a part-per-million effect measurement at high momentum transfers is an extremely complicated task, the forward kinematics seems more favorable. For this kinematics 
a calculation exists [15], where an observation is made that the contribution in the situation where the exchanged photons are nearly real and overtake the external electron kinematics is enhanced as $\ln ^{2}\left(-t / m^{2}\right) \sim 100$, with $m$ the electron mass and $t<0$ the elastic momentum transfer. Making use of the optical theorem, the authors obtained estimates of $B_{n}$ in these kinematics as large as $25-35 \mathrm{ppm}$. The calculation of Ref. [16] that appeared afterward showed that this result is not adequate, since the squared log term can contribute only to the Compton scattering amplitudes with the photon helicity flip. Indeed, this conclusion was confirmed in Refs. [17] and [18], the corrected version of Ref. [15]. It was noticed that the $\ln ^{2}\left(-t / m^{2}\right)$ term should vanish in the forward kinematics owing to gauge invariance, and the leading term is governed by a single (though still large) $\log$ term, $\ln \left(-t / m^{2}\right) \sim 10$. The predictions for $B_{n}$ shifted correspondingly to $\approx-(4-6) \mathrm{ppm}$ and agree with the preliminary data from the HAPPEX experiment [13].

To provide an estimate of $B_{n}$, we use the equivalent photon or quasi-real Compton scattering approximation, which is caused by the hard collinear kinematics responsible for this $\ln ^{2}\left(-t / m^{2}\right)$ enhancement. In this approximation, the leading contribution comes from the kinematic region where both exchanged photons are nearly real. The hadronic tensor is taken at this kinematic point and can be taken out of the integration. For the hadronic tensor, we adopt the most general real Compton scattering amplitude and demostrate that the contribution of the cross section (i.e., photon helicity conserving amplitudes) vanishes in the QRCS approximation. The remaining contributions are related to the photon helicity flip amplitudes. We provide the calculation of $B_{n}$ at low energies, where $\pi N$ intermediate states are expected to be the dominant contributions. In this kinematics, the asymmetry can be related to the pion photoproduction multipoles, and we discuss the relative contributions of different multipoles. At higher energies, we discuss forward kinematics, that is the Regge regime, and note that the combinations of the RCS amplitudes appearing in the expression of $B_{n}$ are related to $2 \pi$ exchange in the $t$ channel. We provide an estimate of such contributions but conclude that these are negligibly small.

The article is organized as follows: in Sec. II we define the kinematics, general $e p$-scattering amplitude, and the observables of interest; in Sec. III the two-photon exchange mechanism and the photons kinematics are studied; the equivalent photons or quasi-real Compton scattering approximation and its implementation for the case of $B_{n}$ is given in Sec. IV; we present our results in Sec. V and conclude with a short summary.

\section{ELASTIC ep-SCATTERING AMPLITUDE}

In this work, we consider the elastic electron-proton scattering process $e(k)+p(p) \rightarrow e\left(k^{\prime}\right)+p\left(p^{\prime}\right)$, for which we define

$$
\begin{aligned}
& P=\frac{p+p^{\prime}}{2}, \quad K=\frac{k+k^{\prime}}{2}, \\
& q=k-k^{\prime}=p^{\prime}-p
\end{aligned}
$$

and choose the invariants ${ }^{2} t=q^{2}<0$ and $v=(P \cdot K) / M$ as the independent variables. $M$ denotes the nucleon mass. They are related to the Mandelstam variables $s=(p+k)^{2}$ and $u=\left(p-k^{\prime}\right)^{2}$ through $s-u=4 M v$ and $s+u+t=2 M^{2}$. For convenience, we also introduce the usual polarization parameter $\varepsilon$ of the virtual photon, which can be related to the invariants $v$ and $t$ (neglecting the electron mass $m$ ):

$$
\varepsilon=\frac{v^{2}-M^{2} \tau(1+\tau)}{v^{2}+M^{2} \tau(1+\tau)}
$$

with $\tau=-t /\left(4 M^{2}\right)$. Elastic scattering of two spin $1 / 2$ particles is described by six independent amplitudes. Three of them do not flip the electron helicity [3],

$$
\begin{aligned}
T_{\text {no flip }}= & \frac{e^{2}}{-t} \bar{u}\left(k^{\prime}\right) \gamma_{\mu} u(k) \\
& \cdot \bar{u}\left(p^{\prime}\right)\left(\tilde{G}_{M} \gamma^{\mu}-\tilde{F}_{2} \frac{P^{\mu}}{M}+\tilde{F}_{3} \frac{\not K P^{\mu}}{M^{2}}\right) u(p),
\end{aligned}
$$

while the other three are electron helicity flipping and thus have, in general, the order of the electron mass $m$ [14]:

$$
\begin{aligned}
T_{\text {flip }}= & \frac{m}{M} \frac{e^{2}}{-t}\left[\bar{u}\left(k^{\prime}\right) u(k) \cdot \bar{u}\left(p^{\prime}\right)\left(\tilde{F}_{4}+\tilde{F}_{5} \frac{\not K}{M}\right) u(p)\right. \\
& \left.+\tilde{F}_{6} \bar{u}\left(k^{\prime}\right) \gamma_{5} u(k) \cdot \bar{u}\left(p^{\prime}\right) \gamma_{5} u(p)\right] .
\end{aligned}
$$

In the one-photon exchange (Born) approximation, two of the six amplitudes match with the electromagnetic form factors,

$$
\begin{aligned}
\tilde{G}_{M}^{\text {Born }}(v, t) & =G_{M}(t), \\
\tilde{F}_{2}^{\text {Born }}(v, t) & =F_{2}(t), \\
\tilde{F}_{3,4,5,6}^{\text {Born }}(v, t) & =0,
\end{aligned}
$$

where $G_{M}(t)$ and $F_{2}(t)$ are the magnetic and Pauli form factors, respectively. For further convenience we define also $\tilde{G}_{E}=\tilde{G}_{M}-(1+\tau) \tilde{F}_{2}$ and $\tilde{F}_{1}=\tilde{G}_{M}-\tilde{F}_{2}$, which in the Born approximation reduce to Sachs electric form factor $G_{E}$ and Dirac form factor $F_{1}$, respectively. For a beam polarized normal to the scattering plane, one can define a single spin asymmetry,

$$
B_{n}=\frac{\sigma_{\uparrow}-\sigma_{\downarrow}}{\sigma_{\uparrow}+\sigma_{\downarrow}},
$$

where $\sigma_{\uparrow}\left(\sigma_{\downarrow}\right)$ denotes the cross sesction for an unpolarized target and for an electron beam spin parallel (antiparallel) to the normal polarization vector, defined as

$$
S_{n}^{\mu}=\left(0, \frac{\left[\vec{k} \times \overrightarrow{k^{\prime}}\right]}{\left|\vec{k} \times \vec{k}^{\prime}\right|}\right),
$$

normalized to $(S \cdot S)=-1$. Similarly, one defines the target normal spin asymmetry $A_{n}$. It has been shown in the early 1970's [7] that such asymmetries are directly related to the

\footnotetext{
${ }^{2}$ In elastic ep scattering, the usual notation for the momentum transfer is $Q^{2}=-q^{2}$, but we prefer the more general notation $t$ to avoid confusion with the incoming and outgoing photon virtualities.
} 
imaginary part of the $T$ matrix. Since the electromagnetic form factors and the one-photon exchange amplitude are purely real, $B_{n}$ obtains its finite contribution to leading order in the electromagnetic constant $\alpha_{\mathrm{em}}$ from an interference between the Born amplitude and the imaginary part of the two-photon exchange amplitude. In terms of the amplitudes of Eqs. (3) and (4), the beam normal spin asymmetry is given by

$$
\begin{aligned}
B_{n}= & -\frac{m}{M} \sqrt{2 \varepsilon(1-\varepsilon)} \sqrt{1+\tau}\left(\tau G_{M}^{2}+\varepsilon G_{E}^{2}\right)^{-1} \\
& \cdot\left[\tau G_{M} \operatorname{Im} \tilde{F}_{3}+G_{E} \operatorname{Im} \tilde{F}_{4}+F_{1} \frac{v}{M} \operatorname{Im} \tilde{F}_{5}\right] .
\end{aligned}
$$

For completeness, we also give here the expression of target normal spin asymmetry ${ }^{3} T_{n}$ in terms of invariant amplitudes:

$$
\begin{aligned}
T_{n}= & \sqrt{2 \varepsilon(1+\varepsilon)} \sqrt{\tau}\left(\tau G_{M}^{2}+\varepsilon G_{E}^{2}\right)^{-1} \\
& \cdot\left[(1+\tau)\left(F_{1} \operatorname{Im} \tilde{F}_{2}-F_{2} \operatorname{Im} \tilde{F}_{1}\right)\right. \\
& \left.+\left(\frac{2 \varepsilon}{1+\varepsilon} G_{E}-G_{M}\right) \frac{v}{M} \operatorname{Im} \tilde{F}_{3}\right]
\end{aligned}
$$

\section{TWO-PHOTON EXCHANGE}

The imaginary part of the two-photon exchange (TPE) graph in Fig. 1 is given by

$$
\begin{aligned}
\operatorname{Im} \mathcal{M}_{2 \gamma}= & e^{2} \int \frac{\left|\vec{k}_{1}\right|^{2} d\left|\vec{k}_{1}\right| d \Omega_{k_{1}}}{2 E_{1}(2 \pi)^{3}} \bar{u}^{\prime} \gamma_{\nu}\left(k_{1}+m\right) \gamma_{\mu} u \\
& \cdot \frac{1}{Q_{1}^{2} Q_{2}^{2}} W^{\mu \nu}\left(w^{2}, Q_{1}^{2}, Q_{2}^{2}\right),
\end{aligned}
$$

where $W^{\mu \nu}\left(w^{2}, Q_{1}^{2}, Q_{2}^{2}\right)$ is the imaginary part of the doubly virtual Compton scattering tensor. $Q_{1}^{2}$ and $Q_{2}^{2}$ denote the virtualities of the exchanged photons in the TPE diagram, and $w$ is the invariant mass of the intermediate hadronic system. We next study the kinematics of the exchanged photons. Neglecting the small electron mass and using the c.m. frame of the electron and proton, one has

$$
Q_{1,2}^{2}=2|\vec{k}|\left|\vec{k}_{1}\right|\left(1-\cos \Theta_{1,2}\right),
$$

with $|\vec{k}|=\left(s-M^{2}\right) / 2 \sqrt{s} \equiv k$ the three-momentum of the incoming (and outgoing) eletron,

$$
\left|\vec{k}_{1}\right|=\sqrt{\left(\frac{s-w^{2}+m^{2}}{2 \sqrt{s}}\right)^{2}-m^{2}}
$$

that of the intermediate electron, and $\cos \Theta_{2}=\cos \Theta \cos \Theta_{1}+$ $\sin \Theta \sin \Theta_{1} \cos \phi$. The kinematically allowed values of the virtualities of the exchanged photons (the restriction is because the intermediate electron is on-shell) are represented by the internal area of the ellipses shown in Fig. 2.

The ellipses are drawn inside a square whose side is defined through the external kinematics $(k)$ and the invariant mass of the intermediate hadronic state $\left(w^{2}\right.$ or $\left.k_{1}\right)$, while the form is

\footnotetext{
${ }^{3}$ Also, $A_{n}$ notation for target normal spin asymmetry exists in the literature.
}

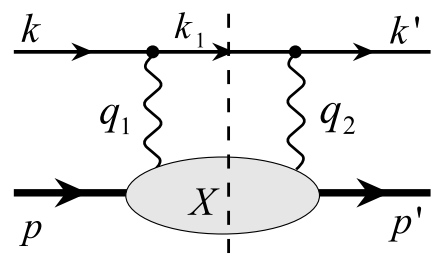

FIG. 1. Two-photon exchange diagram.

determined solely by the scattering angle. Choosing higher values of the mass of the hadronic system $w^{2}<s$ leads to scaling the size of the ellipse by a factor of $\left(s-w^{2}\right) / s-$ $M^{2}$. In the limit $w^{2}=(\sqrt{s}-m)^{2}$, the ellipses shrink to a point at the origin, and both photons are nearly real. This is not a soft photon (IR) singularity, however, since the real photons' energy remains large enough to provide the transition from the nucleon with mass $M$ to the intermediate state $X$ with mass $w$. Instead, the intermediate electron is soft, $k_{1}^{\mu} \approx$ $(m, \overrightarrow{0})$, therefore this kind of kinematics does not lead to an IR divergency, which can occur only if the intermediate hadronic state is the nucleon itself. In the following we are going to study this kinematic situation in more detail.

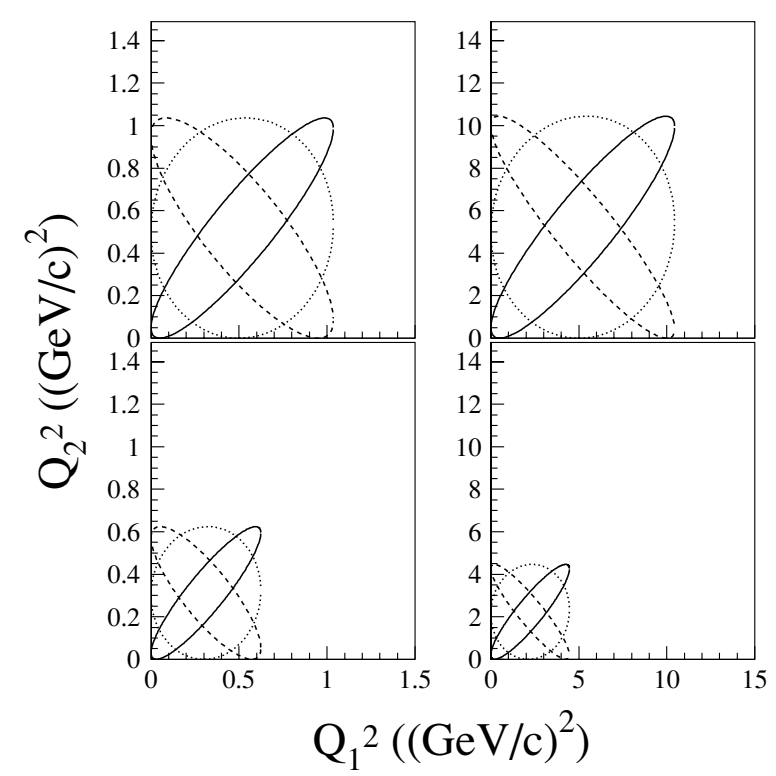

FIG. 2. Kinematically allowed values of the photon vitualities $Q_{1,2}^{2}$. Left, MAMI electron beam energy $E=0.855 \mathrm{GeV}$; right, TJNAF (JLab) energy $E=6 \mathrm{GeV}$. Upper left, different kinematics for the MAMI electron beam energy $E=0.855 \mathrm{GeV}$, for the elastic (nucleon) intermediate state, and three different values of the momentum transfer: $t=-0.2 \mathrm{GeV}^{2}$ (solid ellipse), $t=$ $-0.5 \mathrm{GeV}^{2}$ (dotted ellipse), and $t=-0.9 \mathrm{GeV}^{2}$ (dashed ellipse). Lower left, same external kinematics but with the intermediate hadronic state mass $W=1.232 \mathrm{GeV}$. Upper right, different kinematics for the JLab electron beam energy $E=6 \mathrm{GeV}$, for the elastic (nucleon) intermediate state, and three different values of the momentum transfer: $t=-1 \mathrm{GeV}^{2}$ (solid ellipse), $t=-5 \mathrm{GeV}^{2}$ (dotted ellipse), and $t=-10 \mathrm{GeV}^{2}$ (dashed ellipse). Lower right, the same external kinematics as in the upper right panel, but with the intermediate hadronic state mass $W=2.5 \mathrm{GeV}$. 


\section{QUASI-RCS APPROXIMATION}

We consider the kinematic factors under the integral over the electron phase space of Eq. (10) at the upper limit of the integration over the invariant mass of the intermediate hadronic state, $w \rightarrow \sqrt{s}-m$ :

$$
\frac{\vec{k}_{1}^{2}}{E_{1}} \frac{1}{Q_{1}^{2} Q_{2}^{2}} \sim \frac{1}{4 k^{2} E_{1}} \sim \frac{1}{m} \frac{1}{4 k^{2}} .
$$

In this range of the hadronic mass $w$, the exchanged photons are real, and the contribution of real Compton scattering (RCS) to the $2 \gamma$-exchange graph is enhanced by a factor of $1 / m$ (it is not a singularity, since $B_{n}$ has a factor of $m$ in front).

We next rewrite the contraction of the hadronic and the leptonic tensors in Eq. (10) identically as

$$
\begin{aligned}
l_{\mu \nu} W^{\mu \nu}\left(W^{2}, Q_{1}^{2}, Q_{2}^{2}\right)= & l_{\mu \nu}^{(0)} W^{\mu \nu}(s, 0,0)+l_{\mu \nu}^{(1)} W^{\mu \nu}(s, 0,0) \\
& +l_{\mu \nu}\left[W^{\mu \nu}\left(W^{2}, Q_{1}^{2}, Q_{2}^{2}\right)\right. \\
& \left.-W^{\mu \nu}(s, 0,0)\right],
\end{aligned}
$$

where we expanded the leptonic tensor $l_{\mu \nu}=l_{\mu \nu}^{0}+l_{\mu \nu}^{1}$ with

$$
\begin{aligned}
& l_{\mu \nu}^{0}=m \bar{u}^{\prime} \gamma_{\nu} \gamma_{\mu} u, \\
& l_{\mu \nu}^{1}=\bar{u}^{\prime} \gamma_{\nu} k_{1} \gamma_{\mu} u .
\end{aligned}
$$

In Eq. (13) only the first term does not vanish in the QRCS limit, while the second and third are at least linear in $k_{1}$. In the limit $W^{2} \rightarrow s$, one has $k_{1} \rightarrow m \gamma_{0}$, which is to be compared with the term $m \times 1$ in $l_{\mu \nu}^{0}$. Though at first glance they are of the same order, the additional $\gamma$ matrix picks an extra electron mass $m$ from one of the projectors $k+m$ when performing the summation over electron spins. The quasi-real Compton scattering (QRCS) approximation consists in assuming the first term to be dominant owing to the kinematic enhancement under the integral and in neglecting the second one. In general, this kind of contribution coming from QRCS kinematics will always be present in the full calculation, since the second term in Eq. (13) is constructed in such a way that the resulting integral is regular at the QRCS point. In the following, the QRCS approximation will be used. Hence the hadronic tensor can be taken out of the integration over the electron phase space. The remaining integral is

$$
I_{0}=\int \frac{d^{3} \vec{k}_{1}}{2 E_{1}(2 \pi)^{3}} \frac{1}{Q_{1}^{2} Q_{2}^{2}} .
$$

The result of the integration reads as

$$
I_{0}=\frac{1}{-32 \pi^{2} t}\left[\ln ^{2}\left(\frac{-t}{m^{2}} \frac{E_{\mathrm{thr}}^{2}}{E^{2}}\right)+8 \operatorname{Sp}\left(\frac{E_{\mathrm{thr}}}{E}\right)\right],
$$

where $\operatorname{Sp}(x)$ is the Spence or dilog function, $\operatorname{Sp}(x)=$ $-\int_{0}^{1}(d t / t) \ln (1-x t)$ with $\operatorname{Sp}(1)=\pi^{2} / 6$. In the high-energy limit, $E_{\mathrm{thr}} / E \rightarrow 1$, we recover the result of Ref. [15]. For details, we address the reader to Appendix A. This $\ln ^{2}\left(-t / m^{2}\right)$ factor serves as the justification of the QRCS approximation. In the full result, the term containing $\ln ^{2}\left(-t / m^{2}\right) \sim 100$ contributes along with simple logarithm terms and other terms that do not contain any large logarithms. By studying the QRCS approximation, we show in a model-independent way that the leading term in this expansion is quadratic. Since this leading term is large, we can (under certain kinematic conditions) neglect further terms.

The RCS tensor may be taken, for instance, in the basis of Prange [19] or, equivalently of Berg and Lindner [20],

$$
\begin{aligned}
W_{\mathrm{RCS}}^{\mu \nu}= & \bar{N}^{\prime}\left[\frac{P^{\prime \mu} P^{\prime \nu}}{P^{\prime 2}}\left(B_{1}+\not K B_{2}\right)+\frac{n^{\mu} n^{\nu}}{n^{2}}\left(B_{3}+\not K B_{4}\right)\right. \\
& \left.+\frac{P^{\prime \mu} n^{\nu}-n^{\mu} P^{\prime \nu}}{P^{\prime 2} n^{2}} i \gamma_{5} B_{7}+\frac{P^{\prime \mu} n^{\nu}+n^{\mu} P^{\prime \nu}}{P^{\prime 2} n^{2}} h B_{6}\right] N,
\end{aligned}
$$

with the vectors defined as $P^{\prime}=P-\left(P \cdot K / K^{2}\right) K, n^{\mu}=$ $\varepsilon^{\mu \nu \alpha \beta} P_{\nu} K_{\alpha} q_{\beta}$ such that $\left(P^{\prime} \cdot K\right)=\left(P^{\prime} \cdot n\right)=(n \cdot K)=0$. The amplitudes $B_{i}$ are functions of $v$ and $t$. This form of Compton tensor is convenient because of the simple form of the tensors appearing in Eq. (17).

Before contracting the leptonic and hadronic tensors, we notice that the amplitude $B_{7}$ can contribute only to the invariant amplitude $\tilde{F}_{6}$, since it contains the $\bar{N}^{\prime} \gamma_{5} N$ structure. $\tilde{F}_{6}$ does not contribute at leading order in $m$ to either observable of interest; therefore $B_{7}$ will be neglected in the following. The remaining tensors in Eq. (17) are symmetric in indices $\mu \nu$, and therefore

$$
\operatorname{Im} \mathcal{M}_{2 \gamma}^{\mathrm{QRCS}}=e^{2} m I_{0} \bar{u}^{\prime} u W_{\mathrm{RCS}}^{\mu \nu} g_{\mu \nu} .
$$

Finally, we identify different terms in Eq. (18) with the structures, together with amplitudes $\tilde{F}_{1, \ldots 6}$ parametrizing elastic ep-scattering amplitude in Eqs. (3) and (4), and for the invariant amplitudes for the elastic electron-proton scattering in the QRCS approximation we find

$$
\begin{aligned}
\operatorname{Im} \tilde{G}_{M}^{\mathrm{QRCS}} & =\operatorname{Im} \tilde{F}_{2}^{\mathrm{QRCS}}=\operatorname{Im} \tilde{F}_{3}^{\mathrm{QRCS}}=0, \\
\operatorname{Im} \tilde{F}_{4}^{\mathrm{QRCS}} & =-M t I_{0} \operatorname{Im}\left(B_{1}+B_{3}\right), \\
\operatorname{Im} \tilde{F}_{5}^{\mathrm{QRCS}} & =-M^{2} t I_{0} \operatorname{Im}\left(B_{2}+B_{4}\right) .
\end{aligned}
$$

We obtain for $B_{n}$ in the QRCS approximation

$$
\begin{aligned}
B_{n}= & m t I_{0} \sqrt{2 \varepsilon(1-\varepsilon)} \sqrt{1+\tau}\left(\tau G_{M}^{2}+\varepsilon G_{E}^{2}\right)^{-1} \\
& \times\left\{G_{E} \operatorname{Im}\left(B_{1}+B_{3}\right)+v F_{1} \operatorname{Im}\left(B_{2}+B_{4}\right)\right\} .
\end{aligned}
$$

The result of Eq. (22) is obtained by using only the assumption that the QRCS kinematics dominates the integral in Eq. (10). The combinations of the RCS amplitudes appearing in the final result can be furthermore expressed in terms of the helicity amplitudes of real Compton scattering. With these latter defined as $T_{\lambda_{\gamma}^{\prime}} \lambda_{N}^{\prime}, \lambda_{\gamma} \lambda_{N}, \equiv \varepsilon_{\lambda_{\gamma}^{\prime}}^{\prime * \nu} \varepsilon_{\lambda_{\gamma}^{\prime}}^{\mu} W_{\mu \nu}^{\mathrm{RCS}}$, one has $[21,22]$

$$
\begin{aligned}
B_{1}+B_{3}= & -\frac{1}{\sqrt{-t}}\left(T_{-1-\frac{1}{2}, 1 \frac{1}{2}}+T_{1-\frac{1}{2},-1 \frac{1}{2}}\right) \\
& -\frac{2 M}{M^{4}-s u} T_{1 \frac{1}{2},-1 \frac{1}{2}}, \\
B_{2}+B_{4}= & \frac{2 M}{s-M^{2}} \frac{1}{\sqrt{-t}}\left(T_{-1-\frac{1}{2}, 1 \frac{1}{2}}+T_{1-\frac{1}{2},-1 \frac{1}{2}}\right) \\
& +\frac{2}{M^{4}-s u} \frac{s+M^{2}}{s-M^{2}} T_{1 \frac{1}{2},-1 \frac{1}{2}} .
\end{aligned}
$$


As can be seen, only photon helicity flip amplitudes enter the final result for $B_{n}$ in the QRCS approximation. This is the main result of this work.

\section{RESULTS}

In this section we consider the effect of the QRCS contributions on the beam normal spin asymmetry in different kinematics: low energies ( $\pi N$ intermediate states); high energies and forward angles, i.e. the Regge regime $(2 \pi$ exchange in the $t$-channel); and the hard regime (handbag diagrams and two gluon exchange).

\section{A. Low energies: $\pi \boldsymbol{N}$ multipoles}

Above the pion production threshold, the imaginary part of the RCS helicity amplitudes can be related to the pion photoproduction and electroproduction multipoles. These relations [21,22] for the three amplitudes entering $B_{n}$ read as

$$
\begin{aligned}
\operatorname{Im} T_{-1-\frac{1}{2}, 1 \frac{1}{2}}= & \sin \frac{\Theta}{2} 16 \pi q_{\pi} \sqrt{s} \sum_{k \geqslant 0}(k+1)^{2}\left[\left|A_{k+}\right|^{2}\right. \\
& \left.-\left|A_{(k+1)-}\right|^{2}\right] F\left(-k, k+2,2, \sin ^{2} \frac{\Theta}{2}\right),
\end{aligned}
$$

$$
\begin{aligned}
\operatorname{Im} T_{1-\frac{1}{2},-1 \frac{1}{2}}= & -\sin ^{3} \frac{\Theta}{2} 8 \pi q_{\pi} \sqrt{s} \sum_{k \geqslant 1} \frac{k^{2}(k+1)^{2}(k+2)^{2}}{12} \\
& \times\left[\left|B_{k+}\right|^{2}-\left|B_{(k+1)-}\right|^{2}\right] \\
& \times F\left(-k+1, k+3,4, \sin ^{2} \frac{\Theta}{2}\right), \\
\operatorname{Im} T_{1 \frac{1}{2},-1 \frac{1}{2}}= & \sin ^{2} \frac{\Theta}{2} \cos \frac{\Theta}{2} 8 \pi q_{\pi} \sqrt{s} \sum_{k \geqslant 1} \frac{k(k+1)^{2}(k+2)}{2} \\
& \times \operatorname{Re}\left[B_{k+} A_{k+}^{*}-B_{(k+1)-} A_{(k+1)-}^{*}\right] \\
& \times F\left(-k+1, k+3,3, \sin ^{2} \frac{\Theta}{2}\right),
\end{aligned}
$$

where $q_{\pi}$ is the c.m. pion three-momentum and the hypergeometric function is defined as

$$
F(a, b, c, x)=1+\frac{a b}{c} \frac{x}{1 !}+\frac{a(a+1) b(b+1)}{c(c+1)} \frac{x^{2}}{2 !}+\ldots
$$

We keep only few first multipoles in this infinite series, namely, $A_{0+}, A_{1+}, B_{1+}, A_{2-}, B_{2-}$, which obtain their leading contributions from threshold pion production, $\Delta(1232)$ and $D_{13}(1520)$ resonances. The result for $B_{n}$ reads as

$$
\begin{aligned}
B_{n}= & -8 \pi m q_{\pi} \frac{s t^{2}}{\left(s-M^{2}\right)^{2}} I_{0} \frac{\sqrt{2 \varepsilon(1-\varepsilon)} \sqrt{1+\tau}}{\tau G_{M}^{2}+\varepsilon G_{E}^{2}} \\
& \times\left\{( \frac { E _ { \mathrm { lab } } } { M } F _ { 2 } - F _ { 1 } ) \left[\left|A_{0+}\right|^{2}-\left|A_{1-}\right|^{2}+4\left(\left|A_{1+}\right|^{2}\right.\right.\right.
\end{aligned}
$$

$$
\begin{aligned}
& \left.\left.-\left|A_{2-}\right|^{2}\right)\right]-\frac{6 \sqrt{s}}{M} F_{1} \operatorname{Re}\left(B_{1+} A_{1+}^{*}-B_{2-} A_{2-}^{*}\right) \\
& -\frac{3}{2} \sin ^{2} \frac{\Theta}{2}\left[( \frac { E _ { \mathrm { lab } } } { M } F _ { 2 } - F _ { 1 } ) \left(4\left(\left|A_{1+}\right|^{2}-\left|A_{2-}\right|^{2}\right)\right.\right. \\
& \left.+\left|B_{1+}\right|^{2}-\left|B_{2-}\right|^{2}\right)+4\left(\frac{k}{M} F_{2}-\frac{E}{M} F_{1}\right) \\
& \left.\left.\times \operatorname{Re}\left(B_{1+} A_{1+}^{*}-B_{2-} A_{2-}^{*}\right)\right]\right\} .
\end{aligned}
$$

The helicity multipoles are related to the electromagnetic multipoles $E_{l+,(l+1)-} M_{l+,(l+1)-}$ as

$$
E_{0+}=A_{0+}, \quad M_{1-}=A_{1-},
$$

and for $l \geqslant 1$

$$
\begin{aligned}
E_{l+} & =\frac{1}{l+1}\left[A_{l+}+\frac{l}{2} B_{l+}\right], \\
M_{l+} & =\frac{1}{l+1}\left[A_{l+}-\frac{l+2}{2} B_{l+}\right], \\
E_{(l+1)-} & =-\frac{1}{l+1}\left[A_{(l+1)-}-\frac{l+2}{2} B_{(l+1)-}\right], \\
M_{(l+1)-} & =\frac{1}{l+1}\left[A_{(l+1)-}+\frac{l}{2} B_{(l+1)-}\right] .
\end{aligned}
$$

It is informative to consider the contributions of $E_{0+}$ and $M_{1+}$, which are dominant at low energies. Neglecting other multipoles, we get

$$
\begin{aligned}
B_{n}= & -8 \pi m q_{\pi} \frac{s t^{2}}{\left(s-M^{2}\right)^{2}} I_{0} \frac{\sqrt{2 \varepsilon(1-\varepsilon)} \sqrt{1+\tau}}{\tau G_{M}^{2}+\varepsilon G_{E}^{2}} \\
& \times\left\{\left(\frac{E_{\mathrm{lab}}}{M} F_{2}-F_{1}\right)\left[\left|E_{0+}\right|^{2}+\left|M_{1+}\right|^{2}\right]+\left[\frac{3 \sqrt{s}}{M} F_{1}\right.\right. \\
& \left.\left.+3 \sin ^{2} \frac{\Theta}{2} \frac{(\sqrt{s}-M)^{2}}{2 \sqrt{s} M}\left(\frac{\sqrt{s}+M}{M} F_{2}-F_{1}\right)\right]\left|M_{1+}\right|^{2}\right\} .
\end{aligned}
$$

The first term in the brackets is negative for $E_{\mathrm{lab}} \leqslant M / \kappa \approx$ $0.45 \mathrm{GeV}$ for the proton target, and always negative for the neutron target. Because of the overall minus sign, the beam normal spin asymmetry necessarily obtains a positive contribution from the threshold pion production. Moving toward the $\Delta(1232)$ resonance position, we see that the dominant contribution now comes from the second term. The factor multiplying the $\left|M_{1+}\right|^{2}$ term is always positive for the proton and negative for the neutron. Therefore one expects that the $\Delta$ resonance give a large negative contribution to $B_{n}$ on the proton target and a positive one on the neutron target. We use the photoproduction multipoles of Ref. [23] as input to Eq. (29) and present in Fig. 3 the energy dependence of the beam normal spin asymmetry for the proton target over the resonance region.

This result can be compared with the results of Refs. [10] and [12]. In comparison with the full calculation [10], we find agreement with their findings in the QRCS approximation. In Ref. [12] the authors nominally make the same approximation that we do, keeping the $\ln ^{2}\left(Q^{2} / m^{2}\right)$ and the RCS part of 

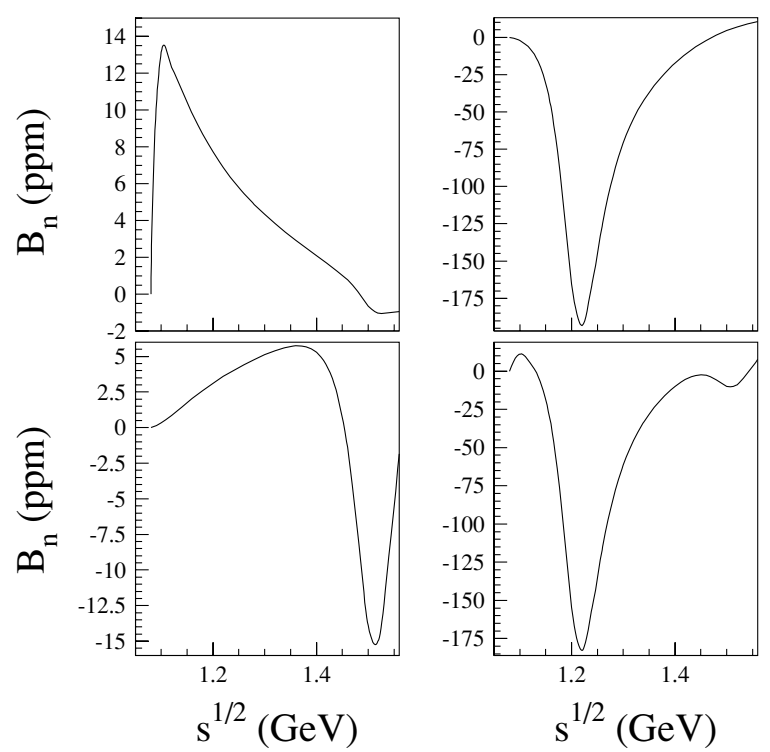

FIG. 3. Beam normal spin asymmetry for the reaction $\vec{e}+p \rightarrow$ $e+p$ in the QRCS approximation at fixed c.m. scattering angle $\Theta_{\text {c.m. }}=120^{\circ}$ as function of the $\pi N$ invariant mass $W_{\pi N}$. The contribution of the $E_{0+}$ multipole (upper left panel), and $E_{1+}$ and $M_{1+}$ (upper right panel), and $E_{2-}$ and $M_{2-}$ (lower left panel) are shown separately. The full result within the QRCS approximation is shown in the lower right panel.

the hadronic tensor only, but arrive at the same sign of the contributions of the $E_{0+}$ and $M_{1+}$ multipoles for the proton. They are furthermore able to describe both low- energy backward-angle data from SAMPLE and intermediate- energy and -angle data from MAMI within the same approximation. It has been shown in Ref. [10] that the QRCS approximation does work well at backward angles (in the sense that it represents the dominant part of the full integration range) but drops short at forward angles, where the exchange of at least one hard virtual photon is important (for very forward angles, see Ref. [15]). Therefore, even if the QRCS approximation did work in the forward regime, one should not take this success too seriously, since the neglected double or single virtual Compton scattering effects are important. We show the angular distributions of $B_{n}$ on the proton target for different values of the electron lab energy in Fig. 4.

In Fig. 5, we display the energy distributions of $B_{n}$ on the neutron over the resonance region.

It is necessary to note that the resonance region does not seem to be favorable for the QRCS approximation. In this approximation, the value of the hadronic amplitude is taken at the largest energy available and is not integrated over the full spectrum. Therefore, if one goes above a resonance position, it is only the tail of the resonance that contributes, which causes the asymmetry to drop quite fast (see the sharp resonance behavior at $\Delta(1232), D_{13}(1520)$ in Fig. 3). In a full calculation, also, intermediate energies of the hadronic system do contribute, and the asymmetry does not have such a sharp energy dependence. So it is preferable to use the QRCS approximation in the region where the energy dependence is rather smooth. Furthermore, the quality of the
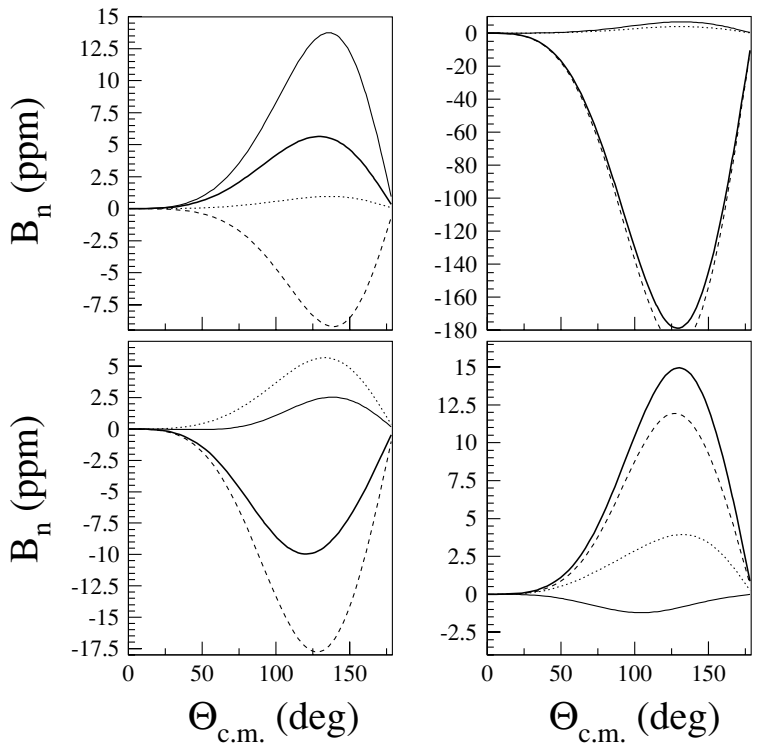

FIG. 4. Beam normal spin asymmetry on the proton as a function of c.m. scattering angle at different values of lab energy: $200 \mathrm{MeV}$ (upper left panel), $300 \mathrm{MeV}$ (upper right panel), $570 \mathrm{MeV}$ (lower left panel), and $855 \mathrm{MeV}$ (lower right panel). The different curves represent $E_{0+}$ multipole contribution (thin solid curves), $E_{1+}$ and $M_{1+}$ contribution (dashed curves), $E_{2-}$ and $M_{2-}$ contribution (dotted curves), and the sum of all (thick solid curves).

QRCS approximation is a function of the scattering angle as well. It works better at backward angles and worse at forward angles. The reason for this is clear: taking the limit of the soft intermediate electron, $k_{1} \approx 0$ and neglecting the dependence of the hadronic and leptonic tensors on $k_{1}$ corresponds to only taking the leading quadratic $\log$ term $\ln 2\left(Q^{2} / \mathrm{m}^{2}\right)$ and neglecting all the remaining terms. The larger $Q^{2}$, the better
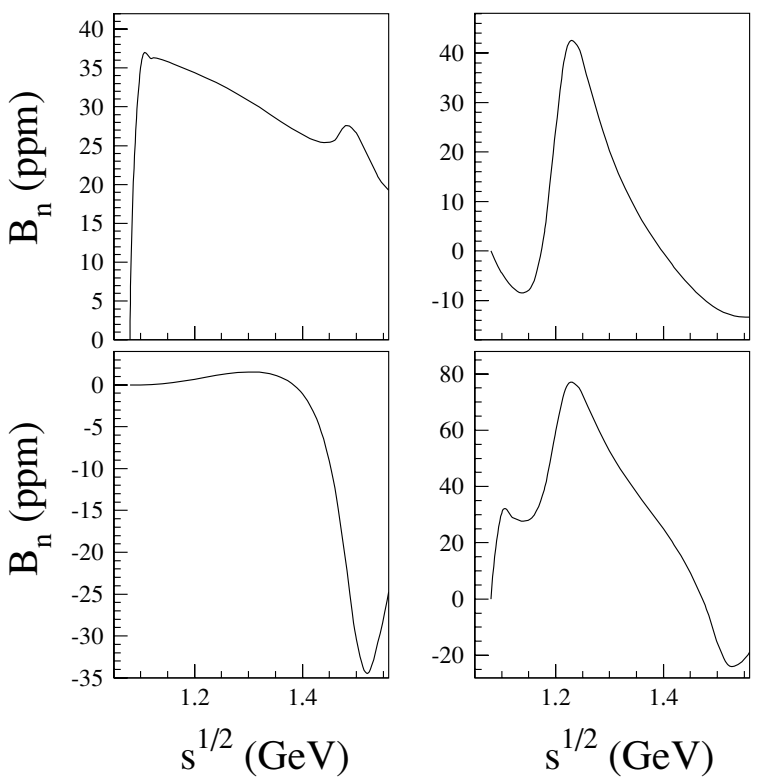

FIG. 5. Beam normal spin asymmetry for the reaction $\vec{e}+n \rightarrow$ $e+n$ in the QRCS approximation as a function of the $\pi N$ invariant mass $W_{\pi N}$. Notation as in Fig. 3. 
the approximation works. This behavior was first observed in Ref. [10]. Instead, results of Ref. [12] suggest that the loop integral in $B_{n}$ is completely dominated by the QRCS region in all the kinematics (backward regime for SAMPLE data and rather forward regime for MAMI data).

\section{B. Regge regime: $2 \pi$ exchange}

The calculation of Ref. [17] estimates $B_{n}$ as

$$
B_{n} \sim \sigma_{\text {tot }} \ln \left(-t / m^{2}\right),
$$

which is based on taking the RCS amplitude in the exact forward limit. If one allows the momentum transfer to be nonzero, however, one obtains the contribution from photon helicity flip amplitudes, which is the result of this work. Though it is logical to expect that these amplitudes be suppressed at low values of $t$, there is a competing factor of $\sim \ln 2\left(Q^{2} / \mathrm{m}^{2}\right)$, which can be of the order of several hundreds. In this subsection we provide an estimate of such a contribution at forward kinematics. The combinations of the RCS amplitudes $B_{1}+B_{3}, B_{2}+B_{4}$ in the Regge regime are known to have the quantum numbers of a scalar isoscalar exchange in the $t$ channel, which was successfully described by $\sigma$-meson [21] or, equivalently, by two-pion exchange in the $t$ channel [24]. Since the effective $\sigma$-meson exchange does not result in a nonzero imaginary part in the $s$ channel, one should use the two-pion exchange mechanism accompanied by a multiparticle intermediate state in the $s$ channel. In this subsection we estimate $B_{1}+B_{3}$ and $B_{2}+B_{4}$ through $\pi N$ and $\rho N$ intermediate state contributions within the minimal Regge model for $\pi$ and $\rho$ photoproduction [25], where a reggeized description of high-energy pion production is obtained by adding the $t$-channel meson exchange amplitude and (in the case of $\gamma p$ reaction) the $s$-channel Born amplitude, which is necessary to ensure gauge invariance. The reggeization procedure naturally leads to the replacement of each $t$-channel Feynman propagator by its Regge counterpart, $1 /\left(t-m_{\pi}^{2}\right) \rightarrow$ $\mathcal{P}_{\pi}^{R}\left[\alpha_{\pi}(t)\right]$, with

$$
\mathcal{P}_{R}^{\pi}=\left(\frac{s}{s_{0}}\right)^{\alpha_{\pi}(t)} \frac{\pi \alpha_{\pi}^{\prime}}{\sin \pi \alpha_{\pi}(t)} \frac{1}{\Gamma\left[1+\alpha_{\pi}(t)\right]},
$$

with $\alpha_{\pi}(t)=\alpha_{\pi}^{\prime}\left(t-m_{\pi}^{2}\right)$ and $\alpha_{\pi}^{\prime}=0.7 \mathrm{GeV}^{-2}$. Gauge invariance requires the $s$-channel piece to be reggeized in the same way, i.e. to be multiplied by $\left(t-m_{\pi}^{2}\right) \mathcal{P}_{\pi}^{R}\left[\alpha_{\pi}(t)\right]$. For compactness, we will use the shorthand [21]

$$
A_{1} \equiv \frac{1}{t}\left[B_{1}+B_{2}+v\left(B_{2}+B_{4}\right)\right]
$$

for the combination of the $B_{i}$ 's that enters the result for the asymmetry. Here we list the results of the calculation of $A_{1}$ :

$$
\begin{aligned}
\operatorname{Im} A_{1}^{\pi N}= & \frac{2 m_{\pi}^{2} C_{\pi}^{2}}{M\left(s-M^{2}\right)}\left(B_{0}^{\pi}-M^{2} A_{0}^{\pi}\right) \\
\operatorname{Im} A_{1}^{\rho N}= & \frac{m_{\rho}^{2} C_{\rho}^{2}}{2 M\left(s-M^{2}\right)}\left(\frac{M^{2}}{s-M^{2}} C_{0}^{\rho}+\frac{s-3 M^{2}+5 m_{\rho}^{2}}{2}\right. \\
& \left.\times B_{0}^{\rho}+m_{\rho}^{2} \frac{s+M^{2}}{2} A_{0}^{\rho}\right)
\end{aligned}
$$

where $C_{\pi}=2 \sqrt{2} \mathrm{Me}\left(f_{\pi N N} / m_{\pi}\right)$ with $f_{\pi N N}^{2} / 4 \pi=0.08$, and $C_{\rho}=2 \sqrt{2} \operatorname{Me}\left[\left(f_{\pi N N} / m_{\pi}\right)\left(f_{\rho \pi \gamma} / m_{\pi}\right)\right]$ with $f_{\rho \pi \gamma}=0.103$ [25]. Where possible, $m_{\pi}$ and $Q^{2}$ were neglected compared with $s, M$, and $m_{\rho}$ in order to simplify the final expression. The scalar integrals $A_{0}, B_{0}$, and $C_{0}$ for both $\pi N$ and $\rho N$ contributions are given in Appendix B.

The result of Eq. (36) leads to a negligibly small contribution to the $B_{n}$, of the order of $10^{-2} \mathrm{ppm}$ for energies between 6 and $45 \mathrm{GeV}$, which is to be compared with $\approx 5 \mathrm{ppm}$ from Ref. [18]. There are three suppression factors that are responsible for this small result. First, the reggeization procedure, leads to suppression in energy $v^{\alpha_{\pi}(t)}$. Second, the amplitude $A_{1}$ is defined as the combination $\frac{1}{t}\left[B_{1}+B_{3}+v\left(B_{2}+B_{4}\right)\right]$, and the singularity $\frac{1}{t}$ in the definition only cancels out if the gauge invariant combination is taken as described in Ref. [25]. Therefore we obtain an additional suppression factor of $t$. The other feature of the result of Eqs. (36) and (A1) is that both contributions are suppressed by factors $m_{\pi}^{2} /\left(s-M^{2}\right)$ and $m_{\rho}^{2} /\left(s-M^{2}\right)$, respectively. In the case of the pion, it is interesting to observe this outcome in view of somewhat the surprising success of the effective field description of the SAMPLE data point on $B_{n}$ without pion contribution. This might give a hint that the pion contribution to $B_{n}$ at low energies is suppressed by the pion mass, if calculated to the same order.

\section{SUMMARY}

In summary, we presented a calculation of the beam normal spin asymmetry. This observable obtains its leading contribution from the imaginary part of the two-photon exchange graph times the Born amplitude and is directly related to the imaginary part of doubly virtual Compton scattering The resulting loop integral obtains a large contribution from the kinematics when both exchanged photons are nearly real and collinear to the external electrons. We adopt the QRCS or equivalent photons approximation, which allows us to take the hadronic part out of the integral and to perform the integration over the electron phase space analytically. For the hadronic part, we use the full real Compton scattering amplitude and show that only photon helicity flip amplitudes contribute in this observable in the QRCS approximation. At low energies, we relate this helicity flipping Compton amplitude to the $\pi N$ multipoles and discuss the contributions of different multipoles.

At high energies and forward scattering angles (Regge regime) we provide an explicite calculation that is due to two-pion exchange in the $t$ channel. The resulting values of the asymmetry are of the order $10^{-2} \mathrm{ppm}$ for the energies in the range 6-45 GeV. We conclude that the double logarithmic enhancement does not dominate $B_{n}$ in the forward regime, since it comes with a helicity flip Compton amplitude that highly suppresses this behavior.

\section{ACKNOWLEDGMENTS}

The author is grateful to Professor M. M. Giannini for continuous support and to Dr. M. J. Ramsey-Musolf and 
D. O'Connell for numerous discussions and for thorough reading of the manuscript. The work was supported by Italian MIUR and INFN, and by U.S. Department of Energy contract DE-FG02-05ER41361.

\section{APPENDIX A: INTEGRALS OVER ELECTRON PHASE SPACE}

In this section we present the calculation of the integrals over electron phase space appearing in the QRCS approximation. First we calculate the scalar integral $I_{0}$,

$$
I_{0}=\int_{0}^{k_{\mathrm{thr}}} \frac{k_{1}^{2} d k_{1}}{2 E_{1}\left(2 \pi^{3}\right)} \int \frac{d \Omega_{k_{1}}}{\left(k-k_{1}\right)^{2}\left(k^{\prime}-k_{1}\right)^{2}},
$$

where the upper integration limit $k_{\text {thr }}$ corresponds to the inelastic threshold (i.e., pion production),

$$
k_{\mathrm{thr}}=\sqrt{\frac{\left(s-\left(M+m_{p} i\right)^{2}\right)^{2}}{4 s}-m^{2}} .
$$

We next introduce integration over the Feynman parameter $1 / a b=\int_{0}^{1} d x /[a+(b-a) x]^{2}$. We chose the polar axis such that $\vec{k}_{1} \cdot(\vec{k}-x \vec{q})=k_{1}|\vec{k}-x \vec{q}| \cos \Theta_{1}$ with $|\vec{k}-x \vec{q}|^{2}=k^{2}+$ $x(1-x) t$ and perform angular integration. We furthermore change integration over $d k_{1}$ to integration over dimensionsless $z=E_{1} / E$,

$$
\begin{aligned}
I_{0}= & \frac{1}{-8 \pi^{2} t} \int_{m / E}^{E_{\mathrm{thr}} / E} \frac{d z}{\sqrt{z^{2}-\frac{m^{2}}{E^{2}}-\frac{4 m^{2}}{t}(1-z)^{2}}} \\
& \times \ln \frac{\sqrt{z^{2}-\frac{m^{2}}{E^{2}}-\frac{4 m^{2}}{t}(1-z)^{2}}+\sqrt{z^{2}-\frac{m^{2}}{E^{2}}}}{\sqrt{z^{2}-\frac{m^{2}}{E^{2}}-\frac{4 m^{2}}{t}(1-z)^{2}}-\sqrt{z^{2}-\frac{m^{2}}{E^{2}}}} .
\end{aligned}
$$

To perform the integration over the electron energy, we follow here the main details of the calculation in Appendix A of Ref. [15]. The result reads as

$$
I_{0}=\frac{1}{-32 \pi^{2} t}\left[\ln ^{2}\left(\frac{-t}{m^{2}} \frac{E_{\mathrm{thr}}^{2}}{E^{2}}\right)+8 \operatorname{Sp}\left(\frac{E_{\mathrm{thr}}}{E}\right)\right],
$$

where $\operatorname{Sp}(x)$ is the Spence or dilog function, $\operatorname{Sp}(x)=$ $-\int_{0}^{1}(d t / t) \ln (1-x t)$ with $\operatorname{Sp}(1)=\pi^{2} / 6$. In the high-energy limit, $E_{\mathrm{thr}} / E \rightarrow 1$, we recover the result of Ref. [15].

\section{APPENDIX B: SCALAR INTEGRALS FOR HELICITY FLIP AMPLITUDE}

The vector and tensor integrals can be reduced to the scalar ones by means of standard methods [26]. The remaining integrals to be calculated are the two-, three-, and four-point scalar integrals. Here we are interested only in the imaginary part of these; therefore there are only three integrals with nonzero imaginary parts: the two-point integral

$$
\begin{aligned}
C_{0}^{\pi} & =\operatorname{Im} \int \frac{d^{4} p_{\pi}}{(2 \pi)^{4}} \frac{1}{p_{\pi}^{2}-m_{\pi}^{2}} \frac{1}{\left(P+K-p_{\pi}\right)^{2}-M^{2}} \\
& =\frac{1}{8 \pi} \frac{\left|\vec{p}_{\pi}\right|}{\sqrt{s}}
\end{aligned}
$$

with

$$
\left|\vec{p}_{\pi}\right|=\sqrt{\frac{\left(s-M^{2}+m_{\pi}^{2}\right)^{2}}{4 s}-m_{\pi}^{2}}
$$

the three-point one

$$
\begin{aligned}
B_{0}^{\pi}= & \operatorname{Im} \int \frac{d^{4} p_{\pi}}{(2 \pi)^{4}} \frac{1}{p_{\pi}^{2}-m_{\pi}^{2}} \frac{1}{\left(k-p_{\pi}\right)^{2}-m_{\pi}^{2}} \\
& \times \frac{1}{\left(P+K-p_{\pi}\right)^{2}-M^{2}} \\
= & -\frac{1}{8 \pi\left(s-M^{2}\right)} \ln \frac{2 E_{\pi}}{m_{\pi}} ;
\end{aligned}
$$

and, finally, the four-point integral

$$
\begin{aligned}
A_{0}^{\pi}= & \operatorname{Im} \int \frac{d^{4} p_{\pi}}{(2 \pi)^{4}} \frac{1}{\left(k-p_{\pi}\right)^{2}-m_{\pi}^{2}} \frac{1}{p_{\pi}^{2}-m_{\pi}^{2}} \\
& \times \frac{1}{\left(k^{\prime}-p_{\pi}\right)^{2}-m_{\pi}^{2}} \frac{1}{\left(P+K-p_{\pi}\right)^{2}-M^{2}} \\
= & \frac{1}{8 \pi Q^{2}\left(s-M^{2}+m_{\pi}^{2}\right)} \\
& \cdot \frac{1}{\sqrt{1+\frac{4 m_{\pi}^{2} E^{2}}{Q^{2} p_{\pi}^{2}}}} \ln \frac{\sqrt{1+\frac{4 m_{\pi}^{2} E^{2}}{Q^{2} p_{\pi}^{2}}}+1}{\sqrt{1+\frac{4 m_{\pi}^{2} E^{2}}{Q^{2} p_{\pi}^{2}}}-1} .
\end{aligned}
$$

These integrals should, however, be reggeized as described in Section V by substituting the Regge propagator instead of the Feynman one. Letting $t_{1}=\left(k-p_{\pi}\right)^{2}$ and $t_{2}=\left(k^{\prime}-p_{\pi}\right)^{2}$ denote the momentum transferred by the pions in the $t$ channel, we have for the reggeized version of scalar integrals

$$
\begin{aligned}
\left(C_{0}^{\pi}\right)^{R}= & \frac{1}{32 \pi^{2}} \frac{p_{\pi}}{\sqrt{s}} \int d \Omega_{\pi}\left(t_{1}-m_{\pi}^{2}\right) \mathcal{P}_{\pi}^{R}\left[\alpha_{\pi}\left(t_{1}\right)\right] \\
& \times\left(t_{2}-m_{\pi}^{2}\right) \mathcal{P}_{\pi}^{R}\left[\alpha_{\pi}\left(t_{2}\right)\right], \\
\left(B_{0}^{\pi}\right)^{R}= & \frac{1}{32 \pi^{2}} \frac{p_{\pi}}{\sqrt{s}} \int d \Omega_{\pi}\left(t_{1}-m_{\pi}^{2}\right) \\
& \times \mathcal{P}_{\pi}^{R}\left[\alpha_{\pi}\left(t_{1}\right)\right] \mathcal{P}_{\pi}^{R}\left[\alpha_{\pi}\left(t_{2}\right)\right], \\
\left(A_{0}^{\pi}\right)^{R}= & \frac{1}{32 \pi^{2}} \frac{p_{\pi}}{\sqrt{s}} \int d \Omega_{\pi} \mathcal{P}_{\pi}^{R}\left[\alpha_{\pi}\left(t_{1}\right)\right] \mathcal{P}_{\pi}^{R}\left[\alpha_{\pi}\left(t_{2}\right)\right] .
\end{aligned}
$$

Similarly, in the case of the $\rho$ exchange in the $s$ channel, the integrals with a nonzero imaginary part are

$$
\begin{aligned}
C_{0}^{\rho} & =\operatorname{Im} \int \frac{d^{4} p_{\rho}}{(2 \pi)^{4}} \frac{1}{p_{\rho}^{2}-m_{\rho}^{2}} \frac{1}{\left(P+K-p_{\rho}\right)^{2}-M^{2}} \\
& =\frac{1}{8 \pi} \frac{\left|\vec{p}_{\rho}\right|}{\sqrt{s}},
\end{aligned}
$$




$$
\begin{aligned}
B_{0}^{\rho}= & \operatorname{Im} \int \frac{d^{4} p_{\rho}}{(2 \pi)^{4}} \frac{1}{p_{\rho}^{2}-m_{\rho}^{2}} \frac{1}{\left(k-p_{\rho}\right)^{2}-m_{\pi}^{2}} \\
& \times \frac{1}{\left(P+K-p_{\rho}\right)^{2}-M^{2}} \\
= & -\frac{1}{8 \pi\left(s-M^{2}\right)} \ln \frac{2 E_{\rho}\left(s-M^{2}\right)}{M m_{\rho}^{2}} \\
A_{0}^{\rho}= & \operatorname{Im} \int \frac{d^{4} p_{\rho}}{(2 \pi)^{4}} \frac{1}{\left(k-p_{\rho}\right)^{2}-m_{\pi}^{2}} \frac{1}{p_{\rho}^{2}-m_{\rho}^{2}} \\
& \times \frac{1}{\left(k^{\prime}-p_{\rho}\right)^{2}-m_{\pi}^{2}} \frac{1}{\left(P+K-p_{\rho}\right)^{2}-M^{2}}
\end{aligned}
$$

$$
\begin{aligned}
& =\frac{1}{8 \pi Q^{2}\left(s-M^{2}+m_{\rho}^{2}\right)} \frac{1}{\sqrt{1+\frac{4 \sigma^{2}}{Q^{2} p_{\rho}^{2}}}} \\
& \quad \times \ln \frac{\sqrt{1+\frac{4 \sigma^{2}}{Q^{2} p_{\rho}^{2}}}+1}{\sqrt{1+\frac{4 \sigma^{2}}{Q^{2} p_{\rho}^{2}}}-1},
\end{aligned}
$$

with

$$
\left|\vec{p}_{\rho}\right|=\sqrt{\frac{\left(s-M^{2}+m_{\rho}^{2}\right)^{2}}{4 s}-m_{\rho}^{2}},
$$

and $\sigma^{2}=E^{2} m_{\rho}^{2}-E E_{\rho}\left(m_{\rho}^{2}-m_{\pi}^{2}\right)+\frac{1}{4}\left(m_{\rho}^{2}-m_{\pi}^{2}\right)^{2}$. If the pion mass is neglected, $\sigma=M m_{\rho}^{2} / 2 s$. The reggeization procedure is the same as for the $\pi N$ intermediate state.

[1] M. K. Jones et al., Phys. Rev. Lett. 84, 1398 (2000); O. Gayou et al., ibid. 88, 092301 (2002); V. Punjabi et al., Phys. Rev. C 71, 055202 (2005); 71, 069902 (2005) [erratum].

[2] L. Andivahis et al., Phys. Rev. D 50, 5491 (1994); M. E. Christy et al., Phys. Rev. C 70, 015206 (2004); I. A. Qattan et al., Phys. Rev. Lett. 94, 142301 (2005) [arXiv:nucl-ex/0410010].

[3] P. A. M. Guichon and M. Vanderhaeghen, Phys. Rev. Lett. 91, 142303 (2003).

[4] L. W. Mo and Y. S. Tsai, Rev. Mod. Phys. 41, 205 (1969); L. C. Maximon and J. A. Tjon, Phys. Rev. C 62, 054320 (2000).

[5] P. G. Blunden, W. Melnitchouk, and J. A. Tjon, Phys. Rev. Lett. 91, 142304 (2003).

[6] Y. C. Chen, A. Afanasev, S. J. Brodsky, C. E. Carlson, and M. Vanderhaeghen, Phys. Rev. Lett. 93, 122301 (2004).

[7] N. F. Mott, Proc. R. Soc. London, Ser. A 135, 429 (1935); A. O. Barut and C. Fronsdal, Phys. Rev. 120, 1871 (1960); A. De Rujula, J. M. Kaplan, and E. De Rafael, Nucl. Phys. B35, 365 (1971).

[8] S. P. Wells et al. (SAMPLE Collaboration), Phys. Rev. C 63, 064001 (2001); F. Maas et al. (MAMI A4 Collaboration), Phys. Rev. Lett. 94, 082001 (2005).

[9] A. Afanasev, I. Akushevich, and N. P. Merenkov, arXiv:hep$\mathrm{ph} / 0208260$.

[10] B. Pasquini and M. Vanderhaeghen, Phys. Rev. C 70, 045206 (2004).

[11] L. Diaconescu and M. J. Ramsey-Musolf, Phys. Rev. C 70, 054003 (2004).
[12] D. Borisyuk and A. Kobushkin, arXiv:nucl-th/0508053.

[13] SLAC E158 Experiment, contact person K. Kumar; G. Cates, K. Kumar, and D. Lhuillier, spokespersons HAPPEX-2 Experiment, JLab E-99-115; D. Beck, spokesperson JLab/G0 Experiment, JLabE-00-6, E-01-116.

[14] M. Gorchtein, P. A. M. Guichon, and M. Vanderhaeghen, Nucl. Phys. A741, 234 (2004).

[15] A. V. Afanasev, N. P. Merenkov, Phys. Lett. B599 48 (2004); Phys. Rev. D 70, 073002 (2004).

[16] M. Gorchtein, arXiv:hep-ph/0505022.

[17] M. Gorchtein, Phys. Rev. C 73, 035213 (2006).

[18] A. V. Afanasev and N. P. Merenkov, hep-ph/0407167 v2.

[19] R. E. Prange, Phys. Rev. 110, 240 (1958).

[20] R. A. Berg and C. N. Lindner, Nucl. Phys. 26, 259 (1961).

[21] A. I. L'vov, V. A. Petrun'kin, and M. Schumacher, Phys. Rev. C 55, 359 (1997).

[22] B. Pasquini, M. Gorchtein, D. Drechsel, A. Metz, and M. Vanderhaeghen, Eur. Phys. J. A 11, 185 (2001).

[23] D. Drechsel, O. Hahnstein, S. S. Kamalov, and L. Tiator, Nucl. Phys. A645, 145 (1999).

[24] D. Drechsel, M. Gorchtein, B. Pasquini, and M. Vanderhaeghen, Phys. Rev. C 61, 015204 (1999).

[25] M. Guidal, J.-M. Laget, and M. Vanderhaeghen, Nucl. Phys. A627, 645 (1997).

[26] G. Passarino and M. Veltman, Nucl. Phys. B160, 151 (1979). 